\title{
Morphology and Life Cycle of Trichinella spiralis Elucidated by Light and Electron Microscopy Techniques
}

\author{
Wieslaw J. Kozek \\ Department of Microbiology and Medical Zoology, Medical Sciences Campus, \\ University of Puerto Rico, San Juan, Puerto Rico.
}

Standard light, scanning, transmission and soft x-ray microscopy techniques were used to elucidate the development and molting pattern of a parasitic nematode, Trichinella spiralis. The newborn larvae were obtained from culture of adults recovered from intestines of rats 5 days after infection; muscle larvae were collected from rats during 24 days after infection, and adult worms, maturing in the intestine of infected rats, were collected at hourly intervals from individual rats up to 36 hours after infection.

The newborn larva, intestinal stages and adults have a similar cuticular structure: superficial horizontal ridges with bars and internal radial striations. The cuticle of the muscle larva is modified into a thick, fairly homogenous, two-layered structure with annular striations and without patent pores of the bacillary bands that are present in the lateral chords of all other stages. Newborn larvae have at least ten functional stichocytes; the stichosome will be fully developed after 16 days of intramuscular development. Sensory structures, observed as modified cilia, were present in the amphids, in cephalic papillae, in some bacillary band cells, and at the end of copulatory appendages. The gender of the mature muscle larva can be identified so by the length of the rectum, by the primordia of the uterus and testis, and the vaginal plate. During intestinal phase, both primordia unite with their respective end organ to form patent ducts. Oocytes and spermatocytes mature concurrently as the respective genital primordia develop into patent male and female gonads, enabling larvae to reach sexual maturity in the intestine at about 30 hours after infection, and insemination to occur immediately afterwards. Larvalinduced transformation of myofibrils of the infected muscle cell into smooth endoplasmic reticulum may be initiated by secretory components of the stichosomes, neurosecretions from the amphids, potential secretions of the bacillary bands or excretions from the intestine. These developmental and morphological features indicate exceptional adaptation of this parasite to maintain its life cycle in nature: the internal development of the newborn larvae continues until the equivalent of fourth stage without molting. A special cuticle is secreted to protect the muscle larvae during its intracellular development and subsequent transition through the stomach's gastric enzymes. The four molts, occurring in rapid succession in the intestine, permit rapid maturation, insemination and larvipostion before the host defenses expel the adults from the intestine. 


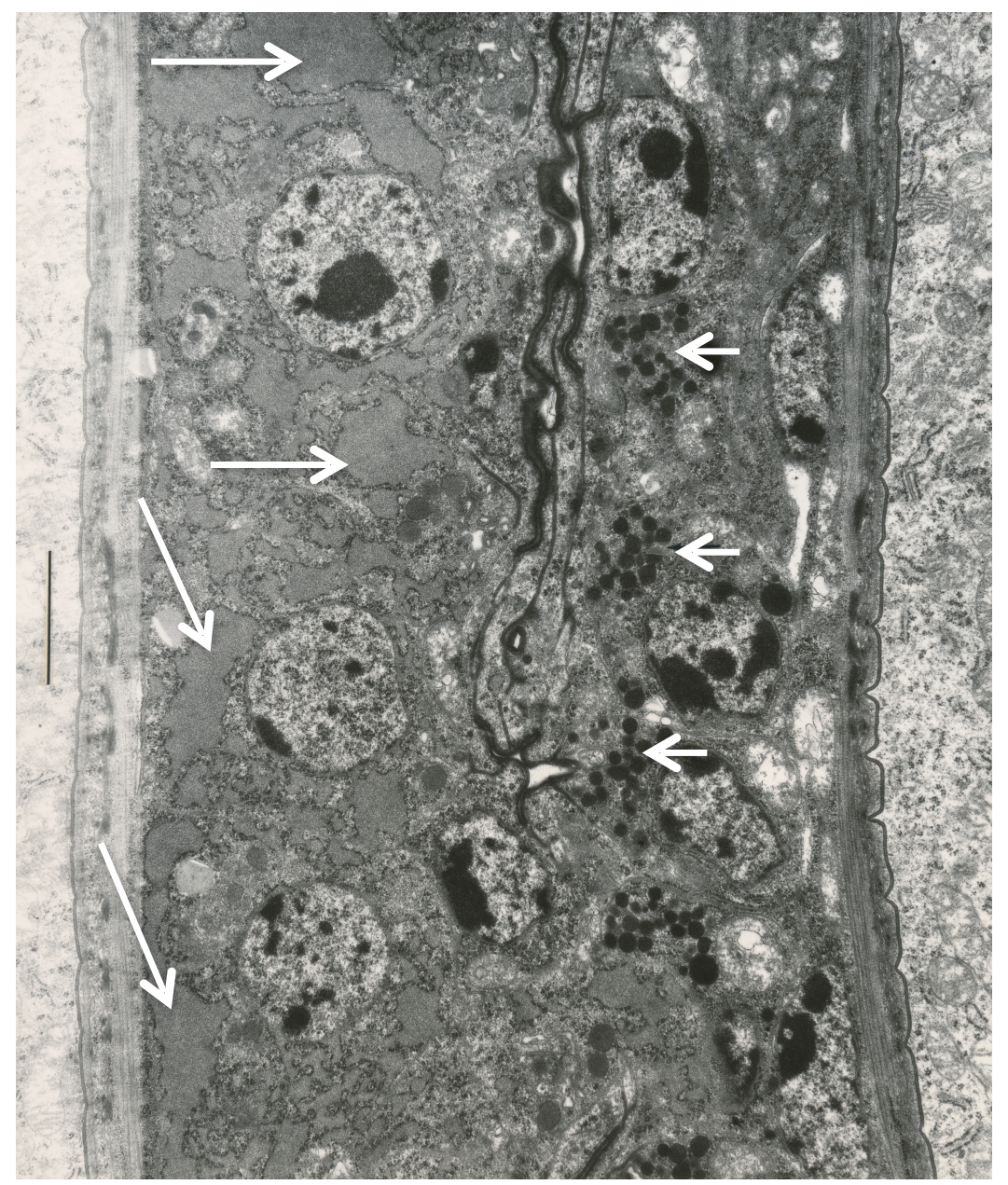

Fig.1. Trichinella spiralis: longitudinal section through developing stichosome of a muscle larva at 14 days of intramuscular development. Note the original stichocytes containing secretory granules (small arrows) still present, and the cisternae (large arrows), within the cytoplasm of new generation of stichocytes, which will form the secretory granules of the mature stichosome. Scale bar $=2 \mu \mathrm{m}$.

\section{References:}

[1] Z Ali Khan, J. Parasit. 62 (1966), p. 284.

[2] LY Wu and A A Kingscote, Can. J. Zool. 35 (1957), p. 207.

[3] The author acknowledges the funding from the U.S. National Institutes of Health, U.S. Department of Energy and RCMI Grant G12MD007600 to the University of Puerto Rico, from the NIH. 